\title{
Predictors of Unrecognized HIV Infection Among Poor and Ethnic Men Who Have Sex with Men in Los Angeles
}

\author{
Sean D. Young $\cdot$ Steven Shoptaw $\cdot$ Robert E. Weiss • \\ Brett Munjas · Pamina M. Gorbach
}

Published online: 31 December 2009

(C) The Author(s) 2009. This article is published with open access at Springerlink.com

\begin{abstract}
This study evaluates associations between unrecognized HIV infection and demographic factors, internalized homonegativity, drug use, and sexual behaviors among HIV positive men who have sex with men (MSM). We analyzed data from 347 HIV positive participants from the Los Angeles site for NIDA's Sexual Acquisition and Transmission of HIV-Cooperative Agreement Program. Participants were HIV positive MSM and MSM/W and predominantly African American (36.0\%) or Latino (38.7\%), and unemployed (82.8\%). Results from a multivariate logistic regression suggest that, compared to HIV positive participants who correctly reported their HIV positive status, being African-American (OR: 9.81, CI: 1.2-77.9) or Latino (OR: 10.92, CI: 1.3-88.4) rather than White, MSM/W rather than MSM (OR: 3.24, CI: 1.09-
\end{abstract}

S. D. Young $(\bowtie)$

Division of Infectious Diseases/Program in Global Health, Department of Medicine, David Geffen School of Medicine at UCLA, 10940 Wilshire Blvd, Suite 1220, Los Angeles, CA 90024, USA

e-mail: sdyoung@mednet.ucla.edu

\section{S. Shoptaw}

Department of Family Medicine, David Geffen School of Medicine at UCLA, Los Angeles, CA, USA

\section{S. Shoptaw}

Department of Psychiatry and Biobehavioral Sciences, David Geffen School of Medicine at UCLA, Los Angeles, CA, USA

R. E. Weiss - B. Munjas

Department of Biostatistics, UCLA School of Public Health, Los Angeles, CA, USA

P. M. Gorbach

Department of Epidemiology, UCLA School of Public Health, Los Angeles, CA, USA
9.62), and having higher homonegativity scores (OR: 1.22, CI: 1.02-1.4) is associated with unrecognized HIV infection, controlling for age, education, and homelessness. Findings provide some immediate evidence to help craft HIV prevention interventions.

Keywords Unrecognized HIV · HIV testing · Homonegativity $\cdot$ Stigma $\cdot$ Awareness of HIV status

\section{Introduction}

As of December 31, 2007, there have been 54,003 reported cases of HIV in Los Angeles County [1]. Men who have sex with men (MSM) and men who have sex with men and women $(\mathrm{MSM} / \mathrm{W})$ have disproportionately high infection rates, accounting for $76.1 \%$ of all Los Angeles County cases [2]. Cases of HIV infection are occurring increasingly and disproportionately in African-American and Latino populations in Los Angeles County [1]. Approximately $25-48 \%$ of HIV + people may be unaware that they are HIV positive (i.e., unrecognized infections) and might therefore unknowingly transmit the virus to others [3-5]. Identifying factors associated with people who are unaware of their HIV-positive status can help to inform prevention efforts toward these populations to both better target persons in need of testing.

One reason for people's lack of awareness of HIV status may be due to stigma. In fact, the Internalized Homonegativity index, a measure of anti-gay attitudes and perceptions of gay-related stigma, has been found to be associated with HIV-positive status and HIV-related risk behaviors within African-American and Latino MSM [6, 7]. Researchers have proposed that high levels of homonegativity and stigma might contribute to African-American 
and Latino MSM engaging in sexual risk behaviors that can transmit HIV [6, 8, 9]. Despite theorized links between homonegativity and sexual risk behaviors, there are no statistical analyses of homonegativity comparing people who are aware of their current HIV status to people who are unaware. Discovering associations between homonegativity and unrecognized HIV may help to guide interventions to increase HIV testing and potentially reduce transmission.

Substance abuse and sexual risk behaviors, such as unprotected sexual intercourse and methamphetamine use, are also associated with HIV infection in African American and Latino MSM communities [10,11], and may be linked to unrecognized HIV infection. Among Latino MSM, substances are reportedly used during sexual intercourse [12], perhaps contributing to sexual risk behavior such as unprotected sexual intercourse when under the influence [13].

The CDC has conducted the majority of studies on unrecognized HIV status because of their access to large populations of HIV positive participants [5, 14]. These studies emphasize the need for studying HIV positive MSM and populations of color as they suggest that being Non-White or MSM is associated with a greater likelihood of unrecognized HIV infection [5]. The present (SATHCAP) dataset includes a large sample of HIV positive participants and therefore has the ability to build on previous studies on unrecognized HIV status by offering (1) an alternative source other than CDC data, and (2) looking at behavioral and psychometric associations with unrecognized HIV status. The present study seeks to assess predictors of unrecognized HIV positive status within a population of HIV positive MSM and MSMW who are poor, urban and of ethnic descent in Los Angeles. Predictors we consider include demographic factors, internalized homonegativity, drug use and sexual risk behaviors.

\section{Methods}

Participants

Participants were MSM or MSM/W from one of the two waves of data collection (797 participants from 2005 to 2006; 795 participants from 2006 to 2008) at the Los Angeles site for NIDA's Sexual Acquisition and Transmission of HIV-Cooperative Agreement Program (SATHCAP) [7].

Sample

The overall SATH-CAP study included participants who were over 18 years of age and MSM or MSM/W who engaged in anal intercourse (AI) in the past 6 months and/ or a male or female drug user (i.e., self-reported use of powder cocaine, crack cocaine, heroin or methamphetamine or any injection drug use). The sample also included sexual partners of these participants. The SATH-CAP sample was recruited using respondent driven sampling [15]. Details of the respondent driven sampling procedures are presented elsewhere [16]. The present study analyzes data on HIV-infected participants from the sample, and includes only MSM and MSM/W as survey skip patterns excused women and heterosexual men from completing the homonegativity questions.

\section{Measures}

The SATH-CAP study used an Audio Computer Assisted Self Interview (ACASI) to collect information regarding: demographic variables such as age, race (broken into African American, White, Latino, and Other), and education; drug use (past 6 months use of powder cocaine, crack cocaine, methamphetamine, heroin and speedballs; sexual risk behaviors, perceptions of HIV risk and sexual "outness" such as comfort in admitting having sex with men to your partner, and the Internalized Homonegativity Inventory (IHNI) [17], an index based on 23 (0-6) point items and 3 subscales (Personal Homonegativity, Gay Affirmation, Morality of Homosexuality) designed to measure homonegativity (or homophobia). Personal Homonegativity included 11 items related to feelings of anxiety, shame, and resentment from being homosexual. Gay Affirmation included 7 items on acceptance and pride related to being homosexual. Morality of Homosexuality included 5 items on moral perceptions of homosexuality. The index and subscores are defined to be the sum of the scores of the individual items. Participants provided biological samples to measure drug use (urine) and HIV (blood). All participants were tested for HIV using rapid blood tests with confirmatory Western Blot.

\section{Data Analysis}

For analysis involving IHNI measures, missing data were handled according to the methods used in previous SATHCAP analyses [18]. Cases missing 3 or fewer items on the IHNI had the missing items imputed with the subject's mean item score to avoid case-wise deletion. Cases with more than 3 missing IHNI items were eliminated from analyses involving IHNI.

The primary outcome of the present analysis was awareness of HIV positive status. We sought to determine associations between awareness/unawareness of HIV positive status and demographic variables, HIV risk behaviors, and homonegativity scores. 
Based on previous methods of measuring unrecognized HIV infection [5, 14, 19, 20], HIV positive status was assessed by confirmation of a lab test. Participants' selfreport of having previously received a positive test result then indicated that they were aware of their HIV positive status. Unrecognized HIV infection was identified based on a biological HIV positive test result and a self-report that the participant either: (1) had previously tested but had not received results, (2) had previously tested negative, or (3) had not previously tested. Psychometric properties of the IHNI in this sample are presented elsewhere [7].

Chi-square tests were used to test independence of HIVpositive status awareness and demographic, drug and sex variables. Logistic regression was used to evaluate the univariate effect of drug and sex variables, and IHNI scores on awareness of HIV positive status. All variables where $P<.1$ were included into a multivariate logistic regression to predict awareness of HIV positive status. Because age and homelessness are important socio-economic variables, they were forced into the multivariate analysis regardless of significance. Female partner knowledge that participants had sex with men was not entered into the multivariate analysis as this measure was only completed by those who had sex with both men and women in the past 6 months $(n=275)$. If similar or related predictors were all significant, a single representative predictor was chosen from the set of predictors.

All scientific and research procedures were overseen by the UCLA Human Subjects Protection Committee and the RAND Institutional Review Board.

\section{Results}

\section{Study Sample}

The total SATH-CAP sample included 1,552 valid participants. The present analysis focuses on a sample of HIV positive MSM and MSM/W. After removing participants with missing data $(n=275)$, female, heterosexual participants and participants who had never had sex $(n=553)$, and HIV-negative men $(n=377)$, the final sample included 347 participants (48.1\% biologically confirmed HIV-positive men out of 722 MSM and MSM/W). Of these 347, 303 participants (87.3\%) were aware of their HIV positive status (i.e., they had previously tested positive) and $41(11.8 \%)$ were coded as being unaware of their HIV infection (i.e., 17 participants had been previously tested but were unaware of their results, 8 had previously tested negative, and 16 had not been tested previously). The 347 HIV+ participants included in these analyses were predominantly African American $(36.0 \%)$ or Latino $(38.7 \%)$ men, unemployed
(82.8\%), earning less than $\$ 500$ each month $(55.8 \%)$, and primarily reporting male-only sex partners in the past 6 months $(74.6 \%)$.

Table 1 shows the results of the univariate logistic regressions using demographic, drug use, sexual behavior, and IHNI variables as predictors of HIV-status-awareness. The items in the internalized homonegativity scale were found to be reliable within the subsample $(\alpha=.9)$. Univariate differences at the $P<.05$ level were observed for cocaine use, and homonegativity. Higher scores on total homonegativity and all three sub-scales (personal, morality, and gay affirmation) were significantly associated with higher unrecognized HIV infection. Differences at the $P<.1$ level were observed for race/ethnicity, gender of sex partner and education.

The results of a multivariate logistic regression incorporating variables from univariate logistic regressions with a threshold of $P<.1$ showed that awareness of unrecognized HIV status was significantly associated with race/ ethnicity, gender of sex partner, and homonegativity, controlling for homelessness, urine screen for cocaine use, education, and age (Table 2). Compared to White participants, African-Americans and Latinos were significantly less likely to be aware of their HIV infection. Compared to MSM, MSM/W were less likely to be aware of their HIV positive status. Participants with greater homonegativity were less likely to be aware of their HIV status. We found no statistically significant differences in awareness based on cocaine usage, age, homelessness, or education in the multivariate analysis.

\section{Discussion}

Results suggest that within a racially and ethnically diverse sample of MSM and MSM/W in Los Angeles, unrecognized HIV infection is associated with race/ethnicity, gender of sexual partner, and homonegativity scores. Race/ethnicity, gender of sexual partner, homonegativity, education, and cocaine use (urine screen) were entered as potential predictors into a multivariate analysis. Race/ethnicity, gender of sexual partner and homonegativity score were significant predictors of correct knowledge of actual HIV status, controlling for age, and homelessness.

Consistent with findings from this large sample, the CDC reported unrecognized infection rates of $48 \%$ in a sample of HIV positive MSM. They found that HIV positive MSM who were Non-White (rather than White), less than 30 (compared to over 30), and surveyed in Los Angeles, New York, Baltimore, or Miami (rather than San Francisco) were less likely to be aware of their HIV positive status [5]. Though parallel, our findings are based 
Table 1 Univariate analysis predicting lack of awareness of HIV positive status (unrecognized infection)

\begin{tabular}{|c|c|c|c|c|c|c|}
\hline & $N$ & $\%$ Unaware & OR & \multicolumn{2}{|c|}{$95 \% \mathrm{CI}$} & $P$-value \\
\hline \multicolumn{7}{|l|}{ Demographic variables } \\
\hline \multicolumn{6}{|l|}{ Age } & .87 \\
\hline$<30$ & 31 & 10 & 1.1 & 0.2 & 4.7 & \\
\hline $30-39$ & 106 & 13 & 1.5 & 0.5 & 4.4 & \\
\hline $40-49$ & 156 & 13 & 1.4 & 0.5 & 4.0 & \\
\hline$\geq 50$ & 54 & 9 & & & & \\
\hline \multicolumn{6}{|l|}{ Race } & .06 \\
\hline White & 66 & 2 & 0.3 & 0.0 & 5.1 & \\
\hline African American & 124 & 16 & 3.8 & 0.5 & 30.3 & \\
\hline Latino & 133 & 14 & 3.3 & 0.4 & 26.3 & \\
\hline Other & 21 & 5 & & & & \\
\hline \multicolumn{6}{|l|}{ Education level } & 0.09 \\
\hline Less than high school (\%) & 86 & 19 & 2.2 & 1.0 & 4.8 & \\
\hline High school & 95 & 11 & 1.2 & 0.5 & 2.7 & \\
\hline More than high school (\%) & 162 & 9 & & & & \\
\hline \multicolumn{6}{|l|}{ Employment status } & .54 \\
\hline Unemployed & 285 & 13 & 1.1 & 0.3 & 3.7 & \\
\hline Part-time & 34 & 6 & 0.5 & 0.1 & 3.0 & \\
\hline Full-time & 25 & 12 & & & & \\
\hline \multicolumn{6}{|l|}{ Income in past month (legal) } & .20 \\
\hline$\$ 0-\$ 500$ & 189 & 15 & 2.6 & 0.8 & 9.0 & \\
\hline$\$ 501-\$ 1,000$ & 102 & 10 & 1.6 & 0.4 & 6.2 & \\
\hline$>\$ 1,000$ & 48 & 6 & & & & \\
\hline \multicolumn{6}{|l|}{ Homeless in past year } & .86 \\
\hline No & 189 & 12 & 0.9 & 0.5 & 1.8 & \\
\hline Yes & 155 & 12 & & & & \\
\hline \multicolumn{6}{|l|}{ Marital status } & .27 \\
\hline Single & 255 & 11 & 0.5 & 0.2 & 1.2 & \\
\hline Married/cohabitating & 35 & 11 & 0.6 & 0.2 & 2.0 & \\
\hline Other & 54 & 19 & & & & \\
\hline \multicolumn{7}{|l|}{ Drug use variables } \\
\hline \multicolumn{6}{|l|}{ Inject drugs_-past 30 days } & .27 \\
\hline No & 299 & 13 & 2.0 & 0.6 & 6.7 & \\
\hline Yes & 44 & 7 & & & & \\
\hline \multicolumn{6}{|l|}{ Inject drugs-ever } & .29 \\
\hline No & 253 & 13 & 1.6 & 0.7 & 3.5 & \\
\hline Yes & 91 & 9 & & & & \\
\hline \multicolumn{6}{|l|}{ Urine drug screen, cocaine } & .04 \\
\hline Negative & 297 & 10 & 0.4 & 0.2 & 1.0 & \\
\hline Positive & 47 & 21 & & & & \\
\hline \multicolumn{6}{|l|}{ Urine drug screen, methamphetamine } & .23 \\
\hline Negative & 298 & 13 & 2.1 & 0.6 & 7.1 & \\
\hline Positive & 46 & 7 & & & & \\
\hline \multicolumn{6}{|l|}{ Urine drug screen, heroin } & .37 \\
\hline Negative & 325 & 12 & 2.5 & 0.3 & 19.4 & \\
\hline Positive & 19 & 5 & & & & \\
\hline \multicolumn{6}{|l|}{ Self report, methamphetamine, last 6 months } & .83 \\
\hline No & 162 & 12 & 1.1 & 0.6 & 2.1 & \\
\hline Yes & 181 & 12 & & & & \\
\hline
\end{tabular}


Table 1 continued

\begin{tabular}{|c|c|c|c|c|c|c|}
\hline & $N$ & $\%$ Unaware & OR & $95 \% \mathrm{CI}$ & & $P$-value \\
\hline Self report, cocaine/crack, last 6 months & & & & & & .37 \\
\hline No & 153 & 14 & 1.4 & 0.7 & 2.6 & \\
\hline Yes & 190 & 11 & & & & \\
\hline Self report, heroin, last 6 months & & & & & & .25 \\
\hline No & 310 & 11 & 0.6 & 0.2 & 1.5 & \\
\hline Yes & 33 & 18 & & & & \\
\hline Self report, speedball, last 6 months & & & & & & .81 \\
\hline No & 313 & 12 & 0.9 & 0.3 & 2.6 & \\
\hline Yes & 30 & 13 & & & & \\
\hline Self report, any drug, last 6 months & & & & & & .64 \\
\hline No & 68 & 10 & 0.8 & 0.3 & 1.9 & \\
\hline Yes & 275 & 12 & & & & \\
\hline \multicolumn{7}{|l|}{ Sexual behaviors } \\
\hline Number of sexual partners-past 6 months & & & & & & .91 \\
\hline None & 53 & 13 & 1.3 & 0.5 & 3.2 & \\
\hline 1 & 51 & 12 & 1.1 & 0.4 & 3.0 & \\
\hline 2 & 51 & 14 & 1.3 & 0.5 & 3.4 & \\
\hline 3 or more & 179 & 11 & & & & \\
\hline Condom use last 6 months & & & & & & .70 \\
\hline No unprotected sex & 163 & 11 & 0.9 & 0.4 & 1.7 & \\
\hline Unprotected sex & 161 & 12 & & & & \\
\hline Sex of sex partners & & & & & & .05 \\
\hline Men, but no sex last 6 months & 53 & 13 & 0.5 & 0.1 & 1.4 & \\
\hline Men only & 249 & 10 & 0.3 & 0.1 & 0.8 & \\
\hline Men and women & 32 & 25 & & & & \\
\hline Female partners know have sex with men* & & & & & & .04 \\
\hline No & 13 & 8 & 0.1 & 0.0 & 0.9 & \\
\hline Yes & 10 & 50 & & & & \\
\hline Male partners know have sex with women* & & & & & & .96 \\
\hline No & 19 & 26 & 1.1 & 0.1 & 12.8 & \\
\hline Yes & 4 & 25 & & & & \\
\hline \multicolumn{7}{|l|}{ IHNI } \\
\hline Unaware & Aware & OR & & $95 \% \mathrm{CI}$ & & $P$-value \\
\hline \multicolumn{7}{|l|}{ IHNI score } \\
\hline Mean (SD) & $53.2(20.8)$ & 1.02 & & 1.01 & 1.04 & .003 \\
\hline \multicolumn{7}{|l|}{ Personal score } \\
\hline Mean (SD) & 23.3(11.6) & 1.03 & & 1.01 & 1.06 & .01 \\
\hline \multicolumn{7}{|l|}{ Morality score } \\
\hline Mean (SD) & $10.1(5.1)$ & 1.07 & & 1.01 & 1.13 & .02 \\
\hline \multicolumn{7}{|l|}{ Gay affirmation score } \\
\hline Mean (SD) & $19.8(8.7)$ & 1.04 & & 1.00 & 1.08 & .03 \\
\hline
\end{tabular}

* Only answered by those who answered that they have had sex with both men and women in the past 6 months, $n=275$

on a sample of MSM and MSM/W who averaged over 40 years of age, reported high homonegativity scores and were very poor. This suggests that poor MSM and MSM/W from communities of color from across the age spectrum are significantly less likely to have accurate knowledge of their current HIV status. Building on past studies of predictors of unrecognized infection, results of this analysis suggest that high homonegativity is a strong predictor of 
Table 2 Multivariate analysis predicting unrecognized infection

\begin{tabular}{|c|c|c|c|c|}
\hline Parameter & $\mathrm{N}$ & OR & 95\% Wald confidence limits & $P$-value \\
\hline \multicolumn{5}{|l|}{ Age } \\
\hline$<30$ & 31 & 1.17 & $(0.2-6.1)$ & 0.85 \\
\hline $30-39$ & 106 & 1.65 & $(0.5-5.4)$ & 0.40 \\
\hline $40-49$ & 156 & 1.40 & $(0.5-4.3)$ & 0.55 \\
\hline$\geq 50$ & 54 & & & \\
\hline \multicolumn{5}{|l|}{ Race } \\
\hline African American & 66 & 9.81 & $(1.2-77.9)$ & 0.03 \\
\hline Latino & 124 & 10.92 & $(1.3-88.4)$ & 0.02 \\
\hline Other & 133 & 2.15 & $(0.1-38.4)$ & 0.60 \\
\hline White & 21 & & & \\
\hline \multicolumn{5}{|l|}{ Education } \\
\hline$<\mathrm{HS}$ & 86 & 1.56 & $(0.7-3.7)$ & 0.31 \\
\hline HS & 95 & 0.59 & $(0.2-1.6)$ & 0.30 \\
\hline$>\mathrm{HS}$ & 162 & & & \\
\hline \multicolumn{5}{|l|}{ Cocaine screen } \\
\hline Positive & 47 & 2.24 & $(0.9-5.7)$ & 0.09 \\
\hline Negative & 297 & & & \\
\hline \multicolumn{5}{|l|}{ Sex of sex partner } \\
\hline Have not had sex last 6 months & 53 & 1.07 & $(0.40-2.8)$ & 0.90 \\
\hline $\mathrm{MSM} / \mathrm{W}$ & 32 & 3.24 & $(1.09-9.62)$ & 0.03 \\
\hline MSM & 249 & & & \\
\hline \multicolumn{5}{|l|}{ Homeless in last year } \\
\hline Homeless & 189 & 1.00 & $(0.5-2.1)$ & 0.99 \\
\hline Not homeless & 155 & & & \\
\hline IHNI score (10 point increase) & 344 & 1.22 & $(1.02-1.4)$ & 0.03 \\
\hline
\end{tabular}

lack of knowledge of HIV status. Future analysis in this area might be used to build on theories of HIV syndemics [21] to determine whether the interaction of psychological and behavioral variables might further reduce recognition of HIV status.

Consistent with prior work suggesting that stigma may reduce perceived risk and willingness to test for stigmatized diseases [6, 22], we found higher homonegativity scores associated with unrecognized HIV positive status. Understanding and addressing associations between homonegativity scores and stigma [23, 24] may improve the design of interventions to facilitate regular HIV testing for MSM communities of color in urban areas. Targeting interventions toward MSM/W, populations of color, and populations with high levels of HIV-related stigma may help to increase testing and treatment and reduce rates of unrecognized HIV.

Study limitations are based on the self-reported nature of findings in a localized population. The convenience sample of poor, ethnic MSM and MSM/W in Los Angeles makes it difficult to generalize findings outside of this community. However, the unique composition of this sample makes it ideal to study to better target prevention and treatment strategies at populations that face disproportionate rates of HIV infections. Second, the stigmatization associated with HIV may have led some participants to underreport being HIV positive, inflating the number of unrecognized infections. Internalized homonegativity scores likely measure only one aspect of stigmatization associated with HIV. Although we included race and education as controls, we were unable to control for other possible variables such as symptoms (people aware of their HIV positive status might have presented with symptoms while unaware participants might not have had symptoms), risk perception (aware participants might have perceived themselves at greater risk and felt a greater need to find their HIV status), and contact with other people with HIV/AIDs (aware participants may have known other people with HIV/AIDS increasing their knowledge about HIV). Finally, the results from the present analysis are based, in part, on a relatively small sample of unrecognized positives. However, because of the scarcity of research on people with unrecognized HIV, we think this is an important contribution to the literature. Additionally, these results are both internally consistent both with the models presented in this study, as well as consistent with past studies on populations of people with unrecognized HIV [5]. 


\section{Conclusions}

Findings show unrecognized HIV infection was associated with being African American or Latino, MSM/W, and having higher homonegativity scores, within a group of HIV positive, poor, predominantly minority, MSM and $\mathrm{MSM} / \mathrm{W}$ in Los Angeles. As efforts increase to devise interventions to encourage routine testing within racial and ethnic MSM, factors addressing homonegativity may provide insight on how best to frame these interventions to be optimally acceptable. The fact remains that significant proportions of MSM from these communities are already infected and further engage in behaviors that spread HIV. Findings provide some immediate evidence that can help craft HIV prevention interventions for these groups.

Acknowledgments The authors with to thank Thomas Coates and Greg Szekeres for feedback on previous versions of this manuscript.

Open Access This article is distributed under the terms of the Creative Commons Attribution Noncommercial License which permits any noncommercial use, distribution, and reproduction in any medium, provided the original author(s) and source are credited.

\section{References}

1. HIV Epidemiology Program Los Angeles County Department of Health Services. HIV/AIDS semi-annual surveillance summary: July 2008. 2008.

2. HIV Epidemiology Program Los Angeles County Department of Health Services. Special data run. Los Angeles. In: Los Angeles County Department of Health Services, editor. 2008.

3. Glynn M, Rhodes P, editors. Estimated HIV prevalence in the United States at the end of 2003 [Abstract]. National HIV Prevention Conference. Atlanta, Georgia; 2005.

4. Branson B, Handsfield H, Lampe M, et al. Revised recommendations for HIV testing of adults, adolescents, and pregnant women in health-care settings. MMWR. 2006;55(RR14):1-17.

5. Centers for Disease Control and Prevention. HIV prevalence, unrecognized infection, and HIV testing among men who have sex with men-Five U.S. cities, June 2004-April 2005. MMWR. 2005;54(24):597-601.

6. Finlayson T. Effects of stigma, sense of community, and selfesteem on the HIV sexual risk behaviors of African-American and Latino men who have sex with men. 2007.

7. Shoptaw S, Weiss R, Munjas B, et al. Homonegativity, substance use, sexual risk behaviors, and HIV-status in poor and ethnic men who have sex with men in Los Angeles. J Urban Health. 2009; 86(Suppl 1):77-92.

8. Carballo-Diéguez A, Miner M, Dolezal C, Rosser BRS, Jacoby S. Sexual negotiation, HIV-status disclosure, and sexual risk behavior among Latino men who use the internet to seek sex with other men. Arch Sex Behav. 2006;35(4):473-81.
9. Poppen P, Reisen C, Zea M, Bianchi F, Echeverry J. Serostatus disclosure, seroconcordance, partner relationship, and unprotected anal intercourse among HIV-positive Latino men who have sex with men. AIDS Educ Prev. 2005;17(3):227-37.

10. Wohl AR, Frye DM, Johnson DF. Demographic characteristics and sexual behaviors associated with metamphetamine use among MSM and Non-MSM diagnosed with AIDS in Los Angeles County. AIDS Behav. 2007;12(5):705-12.

11. O'Leary A, Fisher H, Purcell D, Spikes P, Gomez C. Correlates of risk patterns and race/ethnicity among HIV-positive men who have sex with men. AIDS Behav. 2007;11(5):706-15.

12. Jimenez A. Triple jeaopardy: targeting older men of color who have sex with men. JAIDS-J Acquir Immune Defic Syndr. 2003;33(Suppl 2):S22205.

13. Colfax G, Coates TJ, Husnik MJ, et al. Longitudinal patterns of metamphetamine, popper (amyl nitrate), and cocaine use and high-risk sexual behavior among a cohort of San Francisco men who have sex with men. J Urban Health. 2005;82:i62-i70.

14. Centers for Disease Control and Prevention. Unrecognized HIV infection, risk behaviors, and perceptions of risk among young black men who have sex with men-six U.S. cities, 1994-1998. MMWR. 2002;51(33):733-6.

15. Heckathorn D. Respondent-driven sampling: a new approach to the study of hidden populations. Soc Probl. 1997;44:174-99.

16. Iguchi M, Berry S, Ober A, Jenkins R, Rothenberg R, Heckathorn D. Methodological issues in a dual-core HIV-risk sample (drug users, MSM) recruited through respondent driven sampling. Washington DC: APHA; 2007.

17. Mayfield $\mathrm{W}$. The development of an internalized homonegativity inventory for gay men. J Homosex. 2001;41(2):53-76.

18. Shoptaw S, Weiss RE, Munjas B, et al. Homonegativity, substance use, sexual risk behaviors, and HIV status in poor and ethnic men who have sex with men in Los Angeles. J Urban Health. 2009;86(1):577-92.

19. Weinstock H, Dale M, Linley L, Gwinn M. Unrecognized HIV infection among patients attending sexually transmitted disease clinics. Am J Public Health. 2002;92(2):280-3.

20. MacKellar D, Valleroy L, Secura G, et al. Unrecognized hiv infection, risk behaviors, and perceptions of risk among young men who have sex with men: opportunities for advancing hiv prevention in the third decade of HIV/AIDS. JAIDS-J Acquir Immune Defic Syndr. 2005;38(5):603-14.

21. Stall R, Mills TC, Williamson J, et al. Association of co-occurring psychosocial health problems and increased vulnerability to HIV/AIDS among urban men who have sex with men. Am J Public Health. 2003;93(6):939-42.

22. Young SD, Nussbaum AD, Monin B. Potential moral stigma and reactions to testing for sexually transmitted diseases: evidence for a disjunction fallacy. Personal Soc Psychol Bull. 2007;33(6):78999.

23. Ross MW, Rosser BRS. Measurement and correlates of internalized homophobia: a factor analytic study. J Clin Psychol. 1996;52(1):15-21.

24. Ross MW, Rosser BRS, Neumaier E, Positive Connections Team. The relationship of internalized homonegativity to unsafe sexual behavior in HIV seropositive men who have sex with men. AIDS Educ Prev. 2008;20(6):547-57. 